FRI0410

TGFB PROMOTES FIBROSIS BY MYST1-DEPENDENT EPIGENETIC REGULATION OF AUTOPHAGY

A. Zehender ${ }^{1}$, N.-Y. Lin ${ }^{2}$, A. Stefanica ${ }^{1}$, C.-W. Chen ${ }^{1}$, A. Soare ${ }^{1}$, T. Wohlfahrt ${ }^{1}$, S. Rauber ${ }^{1}$, C. Bergmann ${ }^{1}$, A. Ramming ${ }^{1}$, O. Distler ${ }^{3}$, J. Distler ${ }^{1} .{ }^{1}$ Department of Rheumatology and Internal Medicine (Med3), Friedrich-Alexander-University Erlangen-Nuremberg, Erlangen, Germany; ${ }^{2}$ College of medicine, National Taiwan University (NTU), Taipe, Taiwan, Province of China; ${ }^{3}$ Rheumaklinik, University Hospital Zurich, Zurich, Switzerland

Background: Autophagy is catabolic process allowing cells to degrade unnecessary or dysfunctional cellular organelles. Aberrant activation of autophagy has been also implicated into the pathogenesis of fibrotic diseases. Several stimul present in fibrosis such as pro-fibrotic cytokines are known to activate autophagy. Objectives: The objective of this work is characterise the regulation of autophagy by TGF $\beta$ and analyse whether targeting of autophagy in fibroblasts may prevent their aberrant activation in fibrotic diseases.

Methods: To selectively disable autophagy in fibroblasts we generate $\operatorname{Atg}^{-t^{|l / f|} \mathrm{x}}$ Col1a2;CreER mice. The role of the autophagy was investigated in the model of bleomycin- and T $\beta$ Rlact-induced dermal and pulmonary fibrosis. Overexpression of Myst1 was achieved by adenovirus encoding for Myst1. Collagen release and protein expression were measure by Western blot. Target genes were analysed by RT-PCR. Co-immunoprecipitation and reporter assay were performed to study physical and functional interactions between MYST1 and SMAD3. To monitor the autophagic flux in vitro and in vivo we generate an adenovirus encoding for tandem fluorescent-tagged LC3 (mRFP-EGFP-LC3), defined as reliable autophagy maker.

Results: We provide evidence that transforming growth factor- $\beta$ (TGF $\beta$ ) activates autophagy by an epigenetic mechanism to amplify its profibrotic effects. TGF $\beta$ induces autophagy in fibrotic diseases by SMAD3-dependent downregulation of the H4K16-histoneacetlytransferase MYST1, which controls the expression of core components of the autophagy machinery such as ATG7 and BECLIN1. Activation of autophagy in fibroblasts promotes collagen release and is both, sufficient and required, to induce tissue fibrosis. Forced expression of MYST1 abrogates the stimulatory effects of TGF $\beta$ on autophagy and re-establishes the epigenetic control of autophagy in fibrotic conditions. Interference with the aberrant activation of autophagy inhibits TGF $\beta$-induced fibroblast activation and ameliorates experimental dermal and pulmonary fibrosis. These findings link uncontrolled TGF $\beta$ sig nalling to aberrant autophagy and altered epigenetics in fibrotic diseases and may open new avenues for therapeutic intervention in fibrotic diseases.

Conclusions: We demonstrate that the epigenetic control of autophagy is disturbed by a TGF $\beta$-dependent downregulation of MYST1. The increased activation of autophagy induces fibroblast-to-myofibroblast transition and promotes fibrotic tissue remodelling. Re-expression of MYST1 prevents aberrant autophagy, limits the profibrotic effects of TGF $\beta$ and ameliorates experimental fibrosis. Restoration of the epigenetic control of autophagy might thus be a novel approach to ameliorate fibrotic tissue remodelling.

Disclosure of Interest: A. Zehender: None declared, N.-Y. Lin: None declared, A. Stefanica: None declared, C.-W. Chen: None declared, A. Soare: None declared, T. Wohlfahrt: None declared, S. Rauber: None declared, C. Bergmann: None declared, A. Ramming: None declared, O. Distler Grant/research support from: Actelion, Pfizer, Ergonex, BMS, Sanofi-Aventis, United BioSource Corporation, Roche/Genentech, Medac, Biovitrium, Boehringer Ingelheim, Novartis, 4D Science, Active Biotech, Bayer, Sinoxa, Serodapharm, EpiPharm, GSK, Pharmacyclics and Biogen, Consultant for: Actelion, Pfizer, Ergonex, BMS, Sanofi-Aventis, United BioSource Corporation, Roche/Genentech, Medac, Biovitrium, Boehringer Ingelheim, Novartis, 4D Science, Active Biotech, Bayer, Sinoxa, Serodapharm, EpiPharm, GSK, Pharmacyclics and Biogen, J. Distler Shareholder of: 4D Science, Grant/research support from: Anamar, Active Biotech, Array Biopharma, BMS, Bayer Pharma, Boehringer Ingelheim, Celgene, GSK, Novartis, Sanofi-Aventis, UCB, Consultant for: Actelion, Active Biotech, Anamar, Bayer Pharma, Boehringer Ingelheim, Celgene, Ga-lapagos, GSK, Inventiva, JB Therapeutics, Medac, Pfizer, RuiYi and UCB

DOI: 10.1136/annrheumdis-2018-eular.5891

\section{FRI0411 DUAL PRO-INFLAMMATORY AND ANTI-FIBROTIC ROLE OF IL-17A IN SYSTEMIC SCLEROSIS SKIN}

A.M. Dufour ${ }^{1,2}$, M. Alvarez ${ }^{1,2}$, S. Lemeille ${ }^{1}$, M.-E. Truchetet $^{3}$, N.C. Brembilla ${ }^{1,4}$ C. Chizzolini ${ }^{1,2}$. ${ }^{1}$ Department of Pathology and Immunology, School of Medicine ${ }^{2}$ Immunology and Allergy Department, University Hospital, Geneva, Switzerland ${ }^{3}$ Rheumatology Department, Bordeaux University Hospital, Bordeaux, France; ${ }^{4}$ Dermatology Department, University Hospital and School of Medicine, Geneva, Switzerland

Background: Increased levels of IL-17A have been reported in systemic sclerosis (SSc) which role in fibrogenesis is still debated. ${ }^{1}$ Furthermore, epithelial cells are preferential targets of IL-17A and recent findings suggest that keratinocytes may participate in dysregulated extracellular matrix homeostasis. ${ }^{2}$

Objectives: Our aim was to investigate the interactions between epidermis and dermis in the presence of IL-17A, taking into perspective the fibrotic process. Methods: Primary human keratinocytes were primed with IL-17A and/or TGF- $\beta$ and conditioned-media were used to stimulate healthy donors (HD) and SSc fibroblasts. Alternatively, organotypic cultures of HD full human skin were challenged with these cytokines. Responses were assessed by quantifying inflammatory mediators and type I collagen (Col-I) levels. The factors produced by keratinocytes were identified by a proteomic approach and their contribution was evaluated by neutralisation assays. Changes in gene expression in full human skin after treatment with IL-17A and/or TGF- $\beta$ were analysed by RNA sequencing (RNA-seq)

Results: Unstimulated HD- and SSc-derived keratinocyte-conditioned media (KCM) promoted collagen production by fibroblasts to a similar extent and in a dose-dependent manner. Cytokine array analysis and neutralising assays showed that TGF- $\beta$ was, at least in part, responsible for the pro-fibrotic effect of KCM. Priming of keratinocytes with IL-17A directly decreased Col-I production and significantly reduced $\mathrm{Col}-\mathrm{l}$ induced by TGF- $\beta$ both in SSc and HD fibroblasts. In full human skin, IL-17A promoted pro-inflammatory responses by inducing 2- to 4-fold increase of IL-8, IL-6, MCP-1 and MMP-1 levels, while showing direct antifibrotic effects, as well as decreasing by 2 -fold collagen production triggered by TGF- $\beta(p=0.02)$. RNA-seq revealed that TGF- $\beta$ induced the expression of many collagen genes, while this was not the case for IL-17A. However, IL-17A promoted a pro-inflammatory signature in the skin and strongly downregulated expression of serpin family members, known to be involved in fibrogenesis.

Conclusions: Keratinocytes profoundly influence dermal fibroblast responses which are further modulated in the presence of IL-17A. These data support a role for keratinocytes in the pathogenesis of SSc. IL-17A acts as a potent anti-fibrotic factor in the model of keratinocyte - fibroblast interactions, as well as in the full human skin, promoting pro-inflammatory and anti-fibrotic responses.

\section{REFERENCES:}

[1] Truchetet ME, et al. Arthritis \& Rheumatol 2013;65:1347-56. doi:10.1002/ art.37860

[2] Takahashi T, et al, J Exp Med. 2017 Feb 23. doi:10.1084/jem.20160247

Acknowledgements: Work supported in part by grant 310030-159999 from the Swiss National Science Foundation to CC.

Disclosure of Interest: None declared

DOI: 10.1136/annrheumdis-2018-eular.6784

\section{FRI0412 \\ B CELL DEPLETION AMELIORATES TISSUE FIBROSIS THROUGH REGULATING MACROPHAGE DIFFERENTIATION IN A BLEOMYCIN-INDUCED SYSTEMIC SCLEROSISMODEL MOUSE}

H. Numajiri, A. Yosizaki, T. Fukasawa, S. Ebata, Y. Asano, S. Sato. Department of Dermatology, The University of Tokyo Graduate School of Medicine, Hongo, Bunkyo-ku, Tokyo, Japan

Background: B cells play a critical role in pathogenesis of autoimmune diseases through various functions such as cytokine production and induction of othe immune cell activation. Recent studies have shown the efficacy of B cell depletion therapy with rituximab, a human CD20 chimeric monoclonal antibody, in systemic sclerosis (SSc) patients. However, it still remains unclear why B cell depletion can be effective for SSc.

Objectives: The purpose of this study is to assess the role of B cell depletion in SSc. We evaluated the skin and lung fibrosis of bleomycin (BLM)-induced SSc model mice treated with $B$ cell depletion. Furthermore, we investigated effects of $B$ cell depletion on $T$ cells and macrophages.

Methods: To generate BLM-induced SSc model mice, $200 \mu \mathrm{g}$ of BLM was injected subcutaneously to C57BL/6 mice every other day for 4 weeks. Antimouse CD20 monoclonal antibodies, which can deplete mouse B cells, were also injected every 2 weeks from either one week before (the pre-depletion group) or 2 weeks after (the post-depletion group) BLM treatment. After 4 weeks of BLM treatment, skin and lung fibrosis was assessed histopathologically. To examine the effect of B cell depletion on T cells and macrophages, purified B cells were cultured with $\mathrm{T}$ cells or macrophages and then $\mathrm{T}$ cell cytokine production and macrophage phenotype were analysed.

Results: Skin and lung fibrosis increased in BLM-induced SSc mice. In the coculture experiments, B cells from BLM-induced SSc mice promoted differentiation of $T$ cells producing fibrogenic cytokines such as interleukin-4 compared with contro B cells, while they inhibited regulatory $T$ cell (Treg) differentiation. Skin and lung fibrosis was inhibited in both pre- and post-depletion groups with the inhibition greater in the pre-depletion group than in the post-depletion group. Despite the finding that greater fibrosis remained in the post-depletion group than in the predepletion group, the post-depletion group showed significantly higher frequencies 
of Treg compared with the pre-depletion group. Furthermore, the post-depletion group also exhibited lower fibrogenic cytokine-producing $T$ cell frequencies, suggesting that the change in T cell cytokine production could not account for the more strongly reduced fibrosis observed in the pre-depletion group. Therefore, we examined other immune cell response to B cell depletion. Recent studies have revealed that macrophages are divided into two subtypes: M1 and M2 and that M2 macrophages show fibrogenic effects in SSc. This study showed that macrophages cultured with B cells from BLM-induced SSc mice exhibited enhanced M2 differentiation compared with control B cells. Remarkably, frequencies of M2 macrophage with fibrogenic capacity significantly decreased in the pre-depletion group compared with the post-depletion group, which could account for the more strongly inhibited tissue fibrosis in the pre-depletion group.

Conclusions: Our results indicate that therapeutic effects of $B$ cell depletion on tissue fibrosis exert through regulating macrophage differentiation rather than $T$ cell cytokine production in SSc, first demonstrating that interaction between $\mathrm{B}$ cells and macrophages in development of fibrosis in SSc.

Disclosure of Interest: None declared

DOI: 10.1136/annrheumdis-2018-eular.4655

\section{FRI0413 ARYL HYDROCARBON RECEPTOR EXPRESSION IS ASSOCIATED WITH LUNG INVOLVEMENT IN SYSTEMIC SCLEROSIS}

H. Takei ${ }^{1}$, H. Yasuoka ${ }^{1}$, K. Yoshimoto ${ }^{1,2}$, K. Yamaoka ${ }^{1}$, T. Takeuchi ${ }^{1}{ }^{1}$ Division of Rheumatology, Department of Internal Medicine, Keio University School of Medicine; ${ }^{2}$ Clinical and Translational Research Center, Keio University Hospital, Tokyo, Japan

Background: Systemic sclerosis (SSc) is characterised by autoantibody production, microvascular injury and systemic excessive fibrosis ${ }^{1}$. Genetic and environmental factors are thought to be important for the trigger of development of the disease, however, direct connexion between these factors and pathogenesis of SSc is not yet elucidated ${ }^{23}$. Recent reports showed that environmental toxic pollutants, such as dioxins, play a significant role in the disturbance of immune system and the trigger of autoimmunity through binding aryl hydrocarbon receptor $(A h R)^{4) 5}$. However, little is known about the association between AhR and pathogenesis of SSc yet.

Objectives: To elucidate the association between AhR and the clinical characteristics of SSc.

Methods: Twenty-one patients with SSc who fulfilled 2013 ACR/EULAR classification criteria and 10 healthy controls $(\mathrm{HC})$ were analysed. Peripheral blood mononuclear cells (PBMCs) were isolated from heparinized whole blood by using gradient centrifugation and total RNA was prepared from PBMCs. Expression of Ahr mRNA was detected by quantitative polymerase chain reaction and standardised by mRNA level of $18 S$ ribosomal RNA in each sample. Level of Ahr mRNA in the cells was compared between SSc and HC and also between SSc patients with and without various clinical features.

Results: The proportion of diffuse cutaneous subset (dcSSc) was 33\%. Mean disease duration was $9 \pm 9$ years. The positive proportion of anticentromere antibodies, anti-topoisomerase I antibodies, anti-U1 ribonucleoprotein antibody, antiRNA polymerase III antibody and antinuclear antibody positive without SSc-specific antibodies among 21 patients were 33.3\%, 33.3\%, 4.8\%, 9.5\% and $9.5 \%$, respectively. Antinuclear antibody was negative in $2(9.5 \%)$ patients. Ahr mRNA expression level was tended to be higher in SSc compared to $\mathrm{HC}(1.7 \pm 1.1$ versus $1.2 \pm 0.6, p=0.1)$. Notably, the expression level of $A h R$ mRNA in dc SSc was tended to be higher than that of limited cutaneous SSc $(p=0.15)$, whereas no significant difference was detected between with or without SSc related autoantibodies, vasculopathy such as pulmonary arterial hypertension or digital tip ulcer. Importantly, the expression level of $A h r$ mRNA was significantly higher in patients with interstitial lung disease (ILD) $(n=14)$ than those without $(n=7)(2.0 \pm 1.1$ versus $1.0 \pm 0.4, \mathrm{p}<0.05)$. Furthermore, $A h R$ mRNA expression level was significantly and negatively correlated with DLCO $\%$ predicted $(r=-0.57, p<0.05)$.

Conclusions: Expression level of Ahr mRNA was higher in patients with SSc, especially in SSc patients with ILD. In addition, AhR expression level was correlated with a parameter of pulmonary function test, DLCO\% predicted. These results collectively suggest that AhR possibly plays an important role in the disease process of ILD in SSc.

\section{REFERENCES:}

[1] Nat Rev Rheumatol 2013;9(8):451-64.

[2] Nat. Rev. Rheumatol 2014;10:671-681.

[3] Semin. Immunopathol 2015;37:463-473.

[4] Annu Rev Immunol 2014;32:403-32.

[5] Nature. 2008;453(7191):65-71.

Disclosure of Interest: None declared

DOI: 10.1136/annrheumdis-2018-eular.6594

\section{FRI0414 \\ EVALUATION OF A NOVEL MULTI-ANALYTE ASSAY FOR THE DETECTION OF AUTOANTIBODIES IN THE DIAGNOSIS OF SYSTEMIC SCLEROSIS}

P.L. Meroni ${ }^{1}$, T. Schioppo ${ }^{2}$, F. Ingegnoli ${ }^{2,3}$, M.O. Borghi ${ }^{1,3}$, J.L. Milo ${ }^{4}$, C. Bentow ${ }^{4}$, A. Seaman ${ }^{4}$, G. Valentini ${ }^{5}$, S. Vettori $i^{5}$, P. Di Benedetto ${ }^{6}$, V. Liakouli ${ }^{6}$,

R. Giacomelli ${ }^{6}$, M. Mahler ${ }^{4} .{ }^{1}$ IImmunorheumatology Research Laboratory, Irccs Istituto Auxologico Italiano; ${ }^{2}$ Division of Rheumatology, Asst Pini-CTO;

${ }^{3}$ Department of Clinical Sciences and Community Health, Università degli Studi di Milano, Milan, Italy; ${ }^{4}$ Research and Development, Inova Diagnostics, San Diego, USA; ${ }^{5}$ Department of Clinical and Experimental Medicine, Rheumatology Section, Second University of Naples, Naples; ${ }^{6}$ Department of Rheumatology, University of L'aquila, School of Medicine, L'Aquila, Italy

Background: Systemic sclerosis (SSc) is a chronic autoimmune disease charac terised by vascular changes and progressive fibrosis of skin and various internal organs. In SSc a variety of autoantibodies have been detected which are usefu for the diagnosis and management of the disease. Some of these autoantibodies are well-established tools strongly associated with SSc (e.g. anti-centromere, anti-topoisomerase I, anti-RNA polymerase III). Other autoantibodies are less frequent and/or less-specific for SSc even if potentially useful to better assess disease subsets and prognosis.

Objectives: Our goal was to assess the frequency of SSc-related autoantibodies detected using a novel technology as well as to study the associations between these antibodies and clinical features in an Italian SSc cross sectional cohort.

Methods: Serum samples from 218 consecutive patients with SSc collected at three Italian sites were tested for a variety of autoantibodies (see table 1) using a novel fully automated system utilising bead-based immunoassays (research use only, Inova Diagnostics, San Diego, CA). The Italian cohort included: women 200 (92\%), limited cutaneous SSc (Ic-SSc) 166 (76\%), patients with history of digital ulcers $91(42 \%)$, calcinosis $46(21 \%)$, lung fibrosis $84(39 \%)$, heart involvement 38 $(17.4 \%)$, pulmonary arterial hypertension $20(9 \%)$, and esophageal involvement $138(63.3 \%)$.

Results: The prevalence of antibodies is summarised in the table 1 below. Of note, anti-BICD2, anti-CENP-B, and anti-nucleosome antibodies were significantly associated with Ic-SSc subtype $(p=0.0237, p<0.0001, p=0.0096$, respectively), while anti-Ro60, anti-SSB, anti-Scl-70, and anti-DFS70 antibodies were significantly associated with the diffuse cutaneous SSc (dc-SSc) subtype $(p=0.0102, p<0.0001, p<0.0001$, and $p=0.0032$, respectively). When analysing all antibodies with multivariate analysis, SSc patients showed significant clustering based on antibody profile and clinical phenotype.

\begin{tabular}{|c|c|c|c|c|}
\hline Antibody & Total SSc No. (\%Pos, 95\% Cl) & dc.-SSc No. (\%Pos, 95\% Cl) & IC-SSc No. (\%Pos, 95\% Cl) & $\begin{array}{c}\text { p-value } \\
\text { (dc-SSc vs. Ic-SSc) }\end{array}$ \\
\hline dsDNA & $10(4.6 \%, 95 \%$ Cl $2.5-8.2)$ & $2(3.8 \%, 1.1-1.30 \%)$ & $8(4.8 \%, 2.5-9.2 \%)$ & N.S. \\
\hline RNP & $11(5.0 \%, 95 \% \mathrm{Cl} 2.8-8.8)$ & $3(5.8 \%, 2.0-15.6 \%)$ & $8(4.8 \%, 2.5-9.2 \%)$ & N.S. \\
\hline Ro52 & $32(14.7 \%, 95 \%$ C C 10.6-20.0) & $10(19.2 \%, 10.8-31.9 \%)$ & $22(13.3 \%, 8.9-19.3 \%)$ & N.S. \\
\hline Ro60 & $15(6.9,95 \% \mathrm{Cl} 4.2-11.0)$ & $8(15.4 \%, 8.0-27.5 \%)$ & $7(4.2 \%, 2.1-8.4 \%)$ & 0.0102 \\
\hline ss-B & $7(3.2 \%, 95 \%$ C $11.6-6.5)$ & $7(13.5 \%, 6.7-25.3 \%)$ & $0(0.0 \%, 0.0-2.3 \%)$ & $<0.0001$ \\
\hline Scl-70 & $59(27.1 \%, 95 \% \mathrm{Cl} 21.6-33.3)$ & $39(75.0 \%, 61.8-84.8 \%)$ & $20(12.0 \%, 7.9 \cdot 17.9 \%)$ & $<0.0001$ \\
\hline DF570 & $6(2.8 \%, 95 \%$ C 11.3 .5 .9$)$ & $5(9.6 \%, 4.2 \cdot 20.6 \%)$ & $1(0.6 \%, 0.1-3.3 \%)$ & 0.0032 \\
\hline 10.1 & $2(0.9 \%, 95 \%$ C $10.3-3-3.3)$ & $1(1.9 \%, 0.3 \cdot 10.1 \%)$ & $1(0.6 \%, 0.1-3.3 \%)$ & N.S. \\
\hline CENP-B & $111(50.9 \%, 95 \%$ C $144.3-57.5)$ & $5(9.6 \%, 4.2-20.6 \%)$ & $106(63.9 \%, 56.3-7.70 .8 \%)$ & $<0.0001$ \\
\hline Nucleosome & $38(17.4 \%, 95 \% \mathrm{Cl} 13.0-23.0)$ & $15(28.8 \%, 18.3-42.3 \%)$ & $23(13.9 \%, 9.4-19.9 \%)$ & 0.0096 \\
\hline Ribo-p & $1(0.5 \%, 95 \% \mathrm{Cl} 0.1-2.6)$ & $1(1.9 \%, 0.3-10.1 \%)$ & $0(0.0 \%, 0.0-2.3 \%)$ & N.S. \\
\hline $\mathrm{sm}$ & $0(0.0 \%, 95 \%$ C1 $10.0-1.7)$ & $0(0.0 \%, 0.0-6.9 \%)$ & $0(0.0 \%, 0.0-2.3 \%)$ & N.S. \\
\hline Ku & $6(2.8 \%, 95 \% \mathrm{Cl} 1.3-5.9)$ & $2(3.8 \%, 2.2-13.0 \%)$ & $4(2.4 \%, 0.9-6.0 \%)$ & N.S. \\
\hline PCNA & $1(0.5 \%, 95 \% \mathrm{Cl} 0.1-2.6)$ & $0(0.0 \%, 0.0-6.9 \%)$ & $1(0.6 \%, 0.1-3.3 \%)$ & N.S. \\
\hline RNA POI III & $4(1.8 \%, 95 \% \mathrm{Cl} 0.7-4.6)$ & $2(3.8 \%, 2.2-13.0 \%)$ & $2(1.2 \%, 0.3-4.3 \%)$ & N.S \\
\hline Rpp25 & $2(0.9 \%, 95 \% \mathrm{Cl} 0.3-3.3)$ & $0(0.0 \%, 0.0-6.9 \%)$ & $2(1.2 \%, 0.3-4.3 \%)$ & N.S \\
\hline Rpp38 & $3(1.46,95 \%$ C $10.5-4.0)$ & $0(0.0 \%, 0.0-6.9 \%)$ & $3(1.8 \%, 0.6-5.2 \%)$ & N.S \\
\hline PmScl & $10(4.6 \%, 95 \% \mathrm{Cl} 2.5-8.2)$ & $1(1.9 \%, 0.3-10.1 \%)$ & $9(5.4 \%, 2.9-10.0 \%)$ & N.S. \\
\hline $\mathrm{BICD2}$ & $51(23.4 \%, 95 \%$ Cl 18.3-29.4) & $6(11.5 \%, 5.4-23.0 \%)$ & $45(27.1 \%, 20.9-34.3 \%)$ & 0.0237 \\
\hline MIT3 & $18(8.3 \%, 95 \%$ C1 $5.3-12.7)$ & $2(3.8 \%, 1.1 \cdot 13.0 \%)$ & $16(9.6 \%, 6.0-15.1 \%)$ & N.S. \\
\hline LKM -1 & $0(0.0 \%, 95 \%$ C1 $10.0-1.7)$ & $0(0.0 \%, 0.0-6.9 \%)$ & $0(0.0 \%, 0.0-2.3 \%)$ & N.S. \\
\hline SLA & $1(0.5 \%, 95 \%$ C $10.1-2.6)$ & $0(0.0 \%, 0.0-6.9 \%)$ & $1(0.6 \%, 0.1-3.3 \%)$ & N.S. \\
\hline sp100 & $2(0.9 \%, 95 \%$ C $10.3-3.3 .3)$ & $1(1.9 \%, 0.3-10.1 \%)$ & $1(0.6 \%, 0.1 \cdot 3.3 \%)$ & N.S. \\
\hline $\mathrm{gp} 210$ & $2(0.9 \%, 95 \% \mathrm{Cl} 0.3-3.3)$ & $1(1.9 \%, 0.3-10.1 \%)$ & $1(0.6 \%, 0.1-3.3 \%)$ & N.S. \\
\hline VCP & $0(0.0 \%, 95 \%$ C1 $10.0-1.7)$ & $0(0.0 \%, 0.0-6.9 \%)$ & $0(0.0 \%, 0.0-2.3 \%)$ & N.S. \\
\hline LC-1 & $1(0.5 \%, 95 \% \mathrm{Cl} 0.1-2.6)$ & $0(0.0 \%, 0.0-6.9 \%)$ & $1(0.6 \%, 0.1-3.3 \%)$ & N.S. \\
\hline HK-1 & $5(2.3 \%, 95 \%$ C $11.0-5.3)$ & $2(3.8 \%, 2.2 \cdot 13.0 \%)$ & $3(1.8 \%, 0.6-5.2 \%)$ & N.S \\
\hline Kelch & $2(0.9 \%, 95 \%$ C $10.3-3.3 .3)$ & $0(0.0 \%, 0.0-6.9 \%)$ & $2(1.2 \%, 0.3-4.3 \%)$ & N.S \\
\hline
\end{tabular}

N.S. = not significant

Conclusions: Autoantibodies identified via the novel system in this SSc cohort were found in the expected frequencies and also correlated to clinical features of the patients. The multiparameter approach combined with cluster analysis holds promise for a molecular and more precise stratification of SSc subsets.

Disclosure of Interest: None declared

DOI: 10.1136/annrheumdis-2018-eular.5688 